\title{
FLAME: a library of atomistic modeling environments
}

\author{
Maximilian Amslera, ${ }^{\mathrm{a},}$, Samare Rostami ${ }^{\mathrm{b}}$, Hossein Tahmasbi ${ }^{\mathrm{b}}$, Ehsan Rahmatizad $^{\mathrm{b}}$, Somayeh Faraji $^{\mathrm{b}}$, Robabe \\ Rasoulkhani $^{\mathrm{b}}, \mathrm{S}$. Alireza Ghasemi ${ }^{\mathrm{b}, *}$ \\ ${ }^{a}$ Laboratory of Atomic and Solid State Physics, Cornell University, Ithaca, New York 14853, USA \\ ${ }^{b}$ Department of Physics, Institute for Advanced Studies in Basic Sciences (IASBS), Zanjan 45137-66731, Iran
}

\begin{abstract}
FLAME is a software package to perform a wide range of atomistic simulations for exploring the potential energy surfaces (PES) of complex condensed matter systems. The range of methods include molecular dynamics simulations to sample free energy landscapes, saddle point searches to identify transition states, and gradient relaxations to find dynamically stable geometries. In addition to such common tasks, FLAME implements a structure prediction algorithm based on the minima hopping method (MHM) to identify the ground state structure of any system given solely the chemical composition, and a framework to train a neural network potential to reproduce the PES from ab initio calculations. The combination of neural network potentials with the MHM in FLAME allows a highly efficient and reliable identification of the ground state as well as metastable structures of molecules and crystals, as well as of nano structures, including surfaces, interfaces, and two-dimensional materials. In this manuscript, we provide detailed descriptions of the methods implemented in the FLAME code and its capabilities, together with several illustrative examples.
\end{abstract}

Keywords: structure prediction; neural network; potential energy surface

PROGRAM SUMMARY/NEW VERSION PROGRAM SUMMARY

Program Title: FLAME

Licensing provisions: GPLv3

Programming language: Fortran90, Python

External routines/libraries: BigDFT PSolver [1]4, Spglib [5, MPI, LaPack, Blas

Program summary URL: http://flame-code.org

Program obtainable from: https://github.com/flame-code/ FLAME

in Computer: The program should work on any system with

a F90 compiler. The code has been tested with the Intel and GNU Fortran compilers.

Operating system: Unix/Linux

$R A M$ : several GB

No. of lines in distributed program, including test data, etc.: 231633

Nature of problem: Exploring the potential energy landscapes of complex condensed matter systems, their stationary points, and their global minima.

Solution method: A neural network representation of the potential energy landscape in conjunction with a library of methods to explore its features, most notably the minima hopping approach.

\footnotetext{
* Corresponding authors.

E-mail address: amsler.max@gmail.com, aghasemi@iasbs.ac.ir
}

\section{Introduction}

A wealth of materials properties is intrinsically encoded in the topology of the (free) energy landscape of a condensed matter system. In solids, kinetically persistent atomic structures correspond to local minima on the po'tential energy surface (PES), while the global minimum on the PES represents the ground state structure. In chemical reactions or phase transitions, the reaction coordinate follows the minimal energy pathway (MEP) between two states (local minima) and passes through a first order saddle point. Identifying these stationary points on the PES is of importance in physics, chemistry, and materials science, since the structure of matter fundamentally governs the physical/chemical properties of materials, including crystal phase stability, solubility, optical and transport phenomena, elastic properties, and many more.

Characterizing relevant portions of a PES through atomistic simulations poses two non-trivial tasks, namely (a) finding an accurate representation of the PES and (b) sampling it efficiently:

\section{(a) Representation}

The task of representing the PES requires the mapping of the atomic positions (and the cell vectors for periodic systems) to a scalar total energy based on the underlying atomic interactions. The most accurate methods to compute this energy involve solving in some manner the electronic Schroedinger equation within the BornOppenheimer approximation. Over the last decades, density functional theory (DFT) has evolved as a leading 
method to tackle this challenge due to its convenient accuracy at a relatively moderate computational effort. Nevertheless, the current computer resources limit its application to at most hundreds of atoms when the PES has to be densely sampled through many evaluations, e.g., in structural searches or for molecular dynamics (MD) simulations.

To model larger systems or to accelerate atomistic simulations, alternative methods based on approximate, classical or semi-classical approaches are required, e.g., force fields and tight-binding schemes. In the classical approaches, atomic interactions are approximated by analytical functions justified by the types of the underlying chemical bonds 6 9. Commonly, these interactions include attractive short-range contributions, like stretching, bending, and torsional terms, and non-bonding electrostatic or van der Waals interactions, together with some repulsive terms. Such potentials have to be parameterized and fitted to either experimental or to accurate $a b$ initio data, which limits their application to systems with predetermined compositions and a small set of distinct elements. Nevertheless, modern empirical potentials that include charge transfer algorithms have been applied to a wide range of materials $10-12$.

Recently, with the advent of machine learning (ML) algorithms, there has been a surge in interest for using ML techniques to interpolate the first principles PES by training them on large sets of reference data [13 18. Due to their flexible functional forms, such ML models are capable to reproduce the $a b$ initio results with very high accuracy. However, regions of the configurational space that are not well sampled in the training data set may be poorly described given that ML interatomic potentials are (sophisticated) regression models. Hence, the non-physical form of ML potentials is both a blessing (for interpolation) and a curse (for extrapolation).

\section{(b) Sampling}

Sampling the PES is challenging due to the high dimensionality $D$ of the PES, which is a function of the atomic (and cell) degrees of freedom. For a molecular system with $3 N_{a t}$ atoms, $D$ corresponds to $3 N_{a t}-6$ (the Cartesian coordinates $\mathbf{r}_{1}, \mathbf{r}_{2}, \ldots, \mathbf{r}_{N_{a t}}$ of the atoms, taking into account rotational and translational invariance), while for a crystalline/periodic system $D=3 N_{a t}+3$ (which includes the reduced atomic coordinates $\mathbf{s}_{1}, \mathbf{s}_{2}, \ldots, \mathbf{s}_{N_{a t}}$ with $\mathbf{r}_{i}=h \mathbf{s}_{i}$ and 9 components of the cell vectors $h=\{\mathbf{a}, \mathbf{b}, \mathbf{c}\}$, subject to rotational and translational invariance). Further, the complexity of the PES scales dramatically with the number of degrees of freedom, e.g., the number of local minima on a PES increases exponentially with system size [19], which makes structure prediction challenging for any realistic system 20].

A plethora of packages have been developed in recent years to tackle above tasks separately. AMP [21, PESLearn [22], and TensorMol 23] are only a few examples of codes to generate and train ML models of PESs. They offer optimized workflows to extract structural features and attribute which are used as inputs to train artificial neural network (ANN) or Gaussian process models to accurately reproduce $a b$ initio results. Generally, these codes have to be subsequently coupled to external packages which implement advanced sampling algorithms, like LAMMPS 24] or ASE 25]. Other codes specialized on PES sampling alone with a particular aim at structure prediction range from those based on genetic algorithms (USPEX [26], XtalOpt 27]) and particle swarm optimization (Calypso 28]) to random searches AIRSS [29].

FLAME provides the tools to tackle both challenges within a fully integrated package. In terms of sampling schemes, we have implemented molecular dynamics, saddle point search methods, and, most notably, an efficient structure prediction method based on the MHM. These methods can be coupled to any scheme to evaluate the PES, and we have incorporated interfaces for a range of DFT packages and molecular mechanics tools. At the same time, we have implemented a highly efficient and accurate ANN potential to approximate a PES by training it to $a b$ initio reference data. This synergy of an efficient structure prediction method together with an ANN potential is a particularly powerful feature of FLAME, which significantly accelerates global geometry optimizations and allows the study of larger, increasingly complex and realistic systems.

Beside the ANN as the central technique, FLAME brings along a range of integrated interatomic potentials, including the environment dependent interactive potential (EDIP) 8, Lenosky 9] and Tersoff [7] potentials for silicon. The sampling methods in FLAME can be linked with external codes through sockets, allowing communication across the internet or local UNIX-domain sockets. For this purpose, the i-Pi protocol is implemented, where FLAME acts as a server and the software packages evaluating the PES act as clients 30]. Conversely, FLAME can act as a client as well, conveniently through the i-Pi socket protocol In this way, the methods to evaluate the PES within FLAME can be seamlessly integrated into external codes that provide their own sampling algorithms, e.g., LAMMPS 24 .

This manuscript describes the key features of FLAME and is structured as follows: Sec. 2 briefly describes the input and output file formats of FLAME, Sec. 3 outlines the implementation of the ANN schemes in FLAME, while the relevant sampling algorithms are described in Sec. 4. Each section is accompanied with detailed examples and suggested input parameters, if applicable. In Sec. 5 we review atomic environment descriptors and configurational fingerprints which are implemented in FLAME. We present a brief description of the implemented methods to efficiently calculate the Hartree energy in Sec.6. Finally, we conclude with a summary and an outlook in Sec. 7 . 


\section{Input and Output Files}

FLAME takes no command-line arguments but uses structured input and output files in case-sensitive YAML format for convenient scripting and post-processing. The main input and output files are called flame_in.yaml and flame_log.yaml, respectively, and contain hierarchical keyword-parameter pairs. The main block in flame_in.yaml embeds the most important input key, task, which determines what kind of atomistic simulation to run within the FLAME executable. Further, this block also includes general parameters that describe the system and the computational setup, such as the atomic types, applied pressure, the output verbosity, etc. Essentially, the main block sets up the atomistic modeling environment.

The potential block determines the method used to model the atomic interactions. The associated keyword potential can range from a name of an integrated force field (e.g., $1 \mathrm{j}$ for the Lennard-Jones potential) to the name of an externals software package (e.g., dftb for the DFTB+ package [31, or netsock for network sockets). Additional parameters related to the potential are included here, such as k-points density, cutoff radii, and how to treat long-range electrostatic interactions.

Additional blocks and subblocks can be added to specify the detailed parameters of the simulation. E.g., the method determined in the keyword task itself defines a subblock with its own subparameters. All possible blocks, their keywords, and the associated parameters are printed at the head of the output flame_log.yaml file, thereby allowing a rapid assessment of available and relevant input options. A detailed description of the various keywords can be found in the manual.

The native format of FLAME to handle atomic structure files is based on YAML as well. The default input and output structure filenames are posinp.yaml and posout.yaml, respectively. FLAME brings along a set of convenient python scripts to inter-convert between YAML and other common structure formats, including XYZ, ascii, POSCAR, and many more.

\subsection{Example}

A sample input file for a molecular dynamics simulation with an NVT ensemble of silicon is presented in Fig. 1. The main block determines that a dynamics simulation is to be performed with silicon types of atoms. The employed atomic potential is the Lenosky tight-binding model [9], 1tb. dynamics itself spawns a block, where the method md_method, the time-step dt in fs, and the number of iterations nmd are specified. We set the initial and target temperature to init_temp $=$ temp $=300 \mathrm{~K}$ using the Nose-Hoover (nvt_nose) method with ntherm $=2$ chained thermostats.

Each of the following sections describes in detail the core functionalities of FLAME associated to the task keyword.

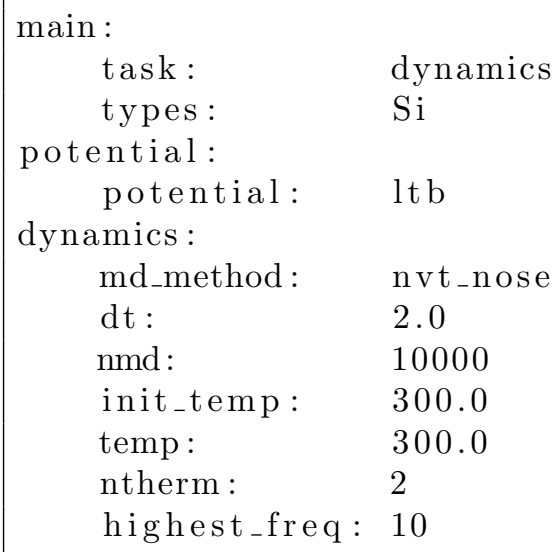

Figure 1: A sample input flame_in.yaml file for an $N V T$ molecular dynamics simulation of a silicon system.

\section{Neural Network Potentials}

In recent years, a new generation of interatomic potentials have been introduced that are based on ML techniques. These potentials are not restricted by a predefined functional form, but are composed of highly flexible multivariate functions with parameters that are optimized during a fitting process, referred to as "training". One type of such an ML potential approach is based on artificial neural networks (ANN) that is inspired by the neural systems in living organisms and imitates how information is passed between neurons through synapses.

In an ANN, the artificial neurons are represented by nodes, which are connected with each other to pass information, the artificial synapses, referred to as edges. Commonly, the nodes are arranged in multiple layers, each containing several nodes. The input data is fed into the ANN through its "input layer" and, after being processed, passes to the next layer, called the "1st hidden layer". The output of this layer is then fed into the next hidden layer, and so on, until the last hidden layer is reached, eventually arriving at the "output layer". The training of an ANN involves fitting the parameters that determine the model by adjusting the weights of the ANN.

Early versions of ANN interatomic potentials used to directly feed the Cartesian coordinates of some or all atoms in a system into the input layer, contained only one node in the output layer, and its value was trained on the total energy of the particular atomic arrangement 32. Two major shortcoming of this approach were quickly recognized: (i) the number of atoms had to remain unchanged for both the training and prediction tasks, hence the ANN wasn't transferable to different stoichiometric conditions, (ii) and the Cartesian coordinates are ill suited as input parameters since they are not invariant under rotation and translation of the structure, while such transformations must preserve the total energy.

In 2007, Behler and Parrinello [13] (BP) introduced a new approach to address above problems. In their method, 
the total energy was expressed as a sum of atomic energies. Each atomic energy $E_{i}$ is thus obtained through an ANN process which is fed with information of the environment of the corresponding atom with index $i$, thereby alleviating problem (i). Problem (ii) was tackled by first mapping the Cartesian coordinates to an array of values describing the local environment of each atom, referred to as an atomic environment descriptor. This transformation is indeed an essential component for any modern ML interatomic potential, and its detailed procedure can significantly affect the performance. Since every part of the BP approach is local, it is less suited for systems in which long range interactions are of importance, e.g., for ionic systems. To improve accuracy, a Coulombic term can be added to the total energy in order to account for the electrostatic interactions 33.

In FLAME, one can select the BP approach by setting approach in block ann to atombased. More details on environment descriptors can be found in Sec. 5.

\subsection{Charge Equilibration via Neural Network Technique}

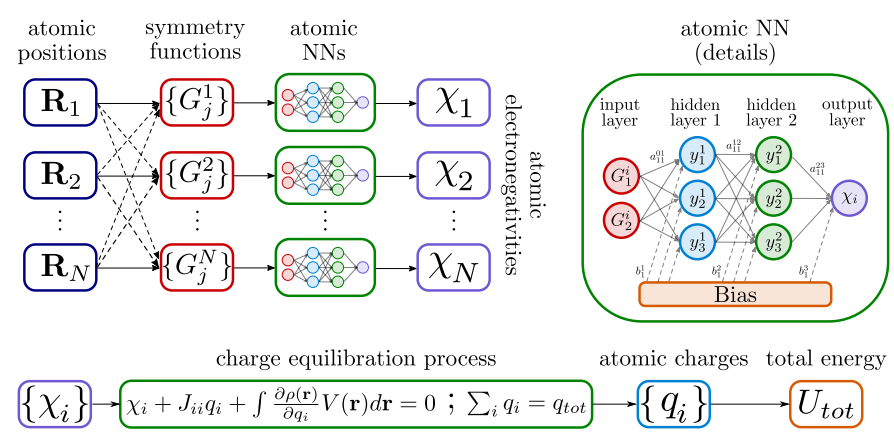

Figure 2: Schematic illustration of the CENT method

In 2015, Ghasemi et al. introduced an alternative approach of employing ML to model atomic interactions. Instead of directly predicting the energy of a system, an ANN process is applied for each atom to predict an environmentdependent atomic electronegativity in the output layer, which in turn is fed into an energy functional [17. This method was coined the "charge equilibration via neural network technique" (CENT) and has been successfully applied to a variety of predominantly ionic materials, including $\mathrm{NaCl}$ [17, $\mathrm{CaF}_{2}$ [34, 35, $\mathrm{ZnO}$ [36, $\mathrm{TiO}_{2}$ [37, $\mathrm{WS}_{2}$ 38] and six-component alkali halide compounds [39]. Overall, errors in physical and chemical quantities investigated in these studies lie well within a few percent with respect to DFT reference values, mostly less than or comparable to the variations among different exchange-correlation functionals employed in DFT calculations. The components of the CENT method, including the high-dimensional neural networks and the charge equilibration process, are depicted in Fig. 2. Here, we give a brief review of the CENT method.

For a system consisting of $N$ atoms, we express the total energy as

$$
\begin{aligned}
U_{t o t}\left(\left\{q_{i}\right\}\right)=\sum_{i=1}^{N}\left(E_{i}^{0}+\chi_{i} q_{i}\right. & \left.+\frac{1}{2} J_{i i} q_{i}^{2}\right) \\
+ & \frac{1}{2} \iint \frac{\rho(\mathbf{r}) \rho\left(\mathbf{r}^{\prime}\right)}{\left|\mathbf{r}-\mathbf{r}^{\prime}\right|} d \mathbf{r} d \mathbf{r}^{\prime},
\end{aligned}
$$

where $E_{i}^{0}$ is the energy of the individual, isolated atom $i$, and $\chi_{i}$ is the environment dependent atomic electronegativity of atom $i$ whose functional dependence is determined by an ANN. $q_{i}$ and $J_{i i}$ are the corresponding atomic charge and the element-dependent atomic hardness [40], respectively, and $\rho(\mathbf{r})$ is the charge density of the system, which, in our implementation, is given by a superposition of spherical Gaussian functions centered at the atomic positions $\mathbf{r}_{i}$, each normalized to the corresponding atomic charge $q_{i}$. For non-periodic systems, Eq. (1) can be rewritten in a simple form:

$$
\begin{array}{r}
U_{\text {tot }}\left(\left\{q_{i}\right\},\left\{\mathbf{r}_{i}\right\}\right)=\sum_{i=1}^{N}\left[\left(E_{i}^{0}+\chi_{i} q_{i}+\frac{1}{2}\left(J_{i i}+\frac{2 \gamma_{i i}}{\sqrt{\pi}}\right) q_{i}^{2}\right]\right. \\
+\sum_{i>j}^{N} q_{i} q_{j} \frac{\operatorname{erf}\left(\gamma_{i j} r_{i j}\right)}{r_{i j}},
\end{array}
$$

where $\gamma_{i j}=\frac{1}{\sqrt{\alpha_{i}^{2}+\alpha_{j}^{2}}}$ and $\alpha_{i}$ are the widths of the Gaussian atomic charge densities, and $r_{i j}$ is the distance between the atoms $i$ and $j$. The energy functional must be minimized with respect to the charge density which, in our scheme, is done by minimizing $U_{t o t}$ with respect to the $q_{i}$ 's, similar to a charge equilibration process. The minimization is performed under the constraint of fixed total charge to a constant value using Lagrange multipliers, which leads to a system of linear equations of the form $\tilde{A} \mathbf{Q}=-\chi$, where $\tilde{A}$ is a $(N+1) \times(N+1)$ matrix, and $\mathbf{Q}$ and $\chi$ are $(N+1)$-dimensional vectors. For non-negative values of $J_{i i}$ 's, it is guaranteed that the matrix of our system of linear equations is non-singular. With this approach, charge can transfer in a long range manner while the total charge of the system is conserved. Note that the atomic charges $q_{i}$ are implicitly environment dependent through the atomic electronegativities $\chi_{i}$.

To solve the system of linear equations using iterative methods, we need the gradient of the total energy with respect to the atomic charges. Differentiating Eq. (1) with respect to $q_{i}$ we obtain the gradient

$$
g_{i}=\frac{\partial U_{t o t}}{\partial q_{i}}=\chi_{i}+J_{i i} q_{i}+g_{i}^{(h)}, \forall i=1, \ldots, N
$$

where $g_{i}^{(h)}$ is the contribution from the Hartree energy and is given by

$$
g_{i}^{(h)}=\int \frac{\partial \rho(\mathbf{r})}{\partial q_{i}} d \mathbf{r} \int \frac{\rho\left(\mathbf{r}^{\prime}\right)}{\left|\mathbf{r}-\mathbf{r}^{\prime}\right|} d \mathbf{r}^{\prime}=\int \frac{\partial \rho(\mathbf{r})}{\partial q_{i}} V(\mathbf{r}) d \mathbf{r} .
$$


The potential function, $V(\mathbf{r})$, can be obtained by solving the Poisson's equation with the appropriate boundary conditions (BC) of the problem. A discussion of the electrostatic methods implemented in FLAME is given in Sec. 6 .

Once the gradient is computed, the system of linear equations can be solved. The constraint of fixed total charge is fulfilled by using the modified gradient given by $g_{i}-\frac{1}{N} \sum_{l=1}^{N} g_{l}$. In the case of free BC and a small number of atoms the system of equations can be solved by means of direct methods, whereas for large systems it is convenient to use an iterative scheme. In contrast, for bulk structures the system of equations is always solved iteratively, irrespective of the system size. Since the system of linear equations is well-conditioned the total number of iterations to reach sufficient convergence rarely exceeds 100 based on extensive tests for small and medium sized systems. Also, it is possible to significantly reduce this number when performing molecular dynamics simulations or local geometry relaxations with relatively small atomic displacements in consecutive time step since the initial guess for the atomic charges can be taken from the converged values obtained in the previous step.

\subsection{Potential Training}

Since ANN ML models do not have a functional form and contain many parameters one may very easily encounter issues due to over-fitting. Therefore, in contrast to usual force fields, one must generate a large number of reference data points ranging from thousands to tens of thousands of configurations. More precisely, the training data must be sufficiently diverse and extensive to prevent over-fitting. In fact, the most challenging task when constructing an accurate and transferable ML potential is generating a suitable reference data set.

We commonly generate such a data set in several steps. First, we start with a small set of configurations that is generated using DFT calculations based on one of the following methods:

i ab initio molecular dynamics simulations starting from different well-known structures at the given composition.

ii random structures that are relaxed to within a very loose tolerance, i.e., by performing only a few iterations as well as using loose input parameters of the $a b$ initio package.

iii elemental substitution in structural prototypes (e.g., such obtain in earlier fitting data sets or online structure repositories) together with an appropriate scaling of the interatomic distances based on the atomic radii.

This small data set is then used to construct a first, approximate CENT potential with limited accuracy. In a next step, this preliminary potential is used in multiple structure prediction runs to sample the PES with various system sizes and starting from different seed configurations. For this purpose we employ the MHM, which not only tries to find the global minimum but also efficiently explores low-lying portions of the PES (see Sec. 4.2.1 for details). In this way, a large number of new structures are generated that can be used to extend the initial training data set. Since the approximate potential trained to the first, small training set can produce nonphysical structures, we have to exclude them and filter for structures with, e.g., unreasonable bond lengths. We further screen the data set for configurations that are too similar to each other by using distances of atomic environment descriptors or structural fingerprints in order to retain a high structural diversity. Finally, DFT calculations are performed on the new configurations to update the training data set and construct a more accurate CENT potential.

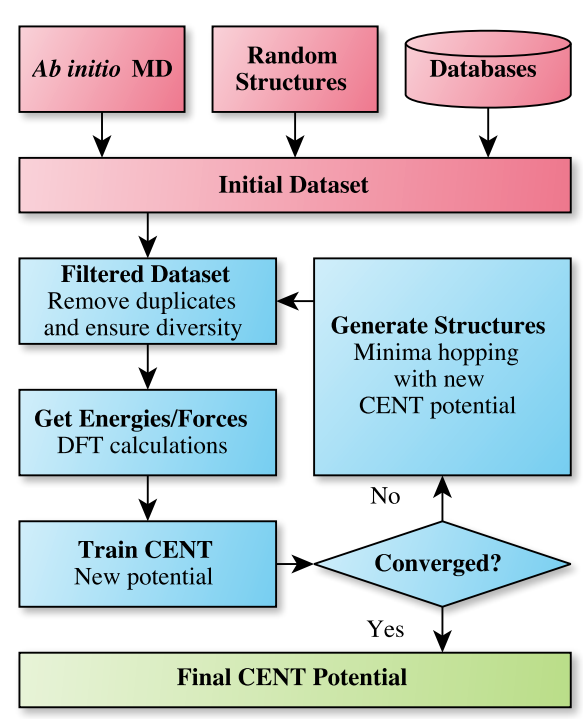

Figure 3: Flowchart of the CENT training procedure.

In practice, we repeat this process of refining the reference data several times until the training set is sufficiently large and contains diverse structures to reach the desired accuracy and reliability of the resulting CENT potential. All the steps involved in the procedure above are fully implemented in FLAME, i.e., training an ANN potential, excluding similar structures, performing MHM or MD runs to generate new structures, etc. Fig. 3 shows a flowchart of the iterative CENT training algorithm.

\subsection{Example}

As a model system to illustrate the performance of CENT, we train an interatomic potential for magnesium oxide $(\mathrm{MgO})$ in the present study. $\mathrm{MgO}$ is a strongly ionic material which crystallizes in the rock-salt structure with a wide band gap of about $7.8 \mathrm{eV}$, and is predominantly used as a refractory material due to its high thermal stability. Our ANN potential for $\mathrm{MgO}$ is then used to demonstrate 
main :

$\begin{array}{ll}\text { task: } & \text { ann } \\ \text { types: } & \mathrm{MgO}\end{array}$

ann :

subtask: $\quad$ train

approach : cent 1

optimizer: rivals

nstep_opt : $\quad 15$

nconf_rmse: $\quad 400$

ampl_rand: $\quad 0.02$

symfunc: only_calculate

potential:

potential: ann

ewald :
ewald :
False

Figure 4: A sample input flame_in.yaml file to train an ANN model within the CENT scheme. The main block determines the task (ann) and the chemical system (here, $\mathrm{Mg}-\mathrm{O}$ ). The ann block determines the subtask to be performed, namely training, together with a range of parameters: the cent1 scheme is specified for the ANN approach using the Kalman optimizer as implemented by rivals 41 with nstep_opt $=15$ optimization steps (epochs), while nconf_rmse $=400$ structures are randomly selected from the reference set as training data. The keyword ampl_rand sets the amplitude of the random ANN weights during initialization, and the symmetry functions (symfunc) are computed on the fly (only_calculate) without reading or writing them to disk. The details of the interatomic potential is given in the potential block. A description of all keywords and their associated parameters can be found in the manual.

some of the key capabilities of FLAME throughout this manuscript.

We generate the reference data set as described in Sec. 3.2 and include both clusters and periodic bulk structures. In addition to stoichiometric $\mathrm{MgO}$ we also incorporate a significant fraction of non-stoichiometric compositions. Hence, the resulting potential is well suited to model neutral as well as charged systems, including clusters, nano structures, and crystalline solids. To train the ANN weight parameters in the CENT potential we split the reference data set into a training part and a validation part. By carefully monitoring the root mean square error (RMSE) of the CENT predictions for the validation data set we can benchmark the performance and easily detect issues arising from over-fitting. A sample input flame_in.yaml file for the CENT fitting process is shown in Fig. 4, and Tab. 1 contains the detailed makeup of the reference data set.

Fig. 5(a) shows the convergence of the RMSE with respect to the number of training epochs for all structures in the training and validation data sets. The subfigures 5(b)(d) compare the convergence across structures with different types of BC and compositions. Overall, the behavior only depends weakly on the selected subset of training data. The largest RMSE is observed when considering only bulk structures, which is however not surprising give that we included much fewer bulk configurations compared to clusters.
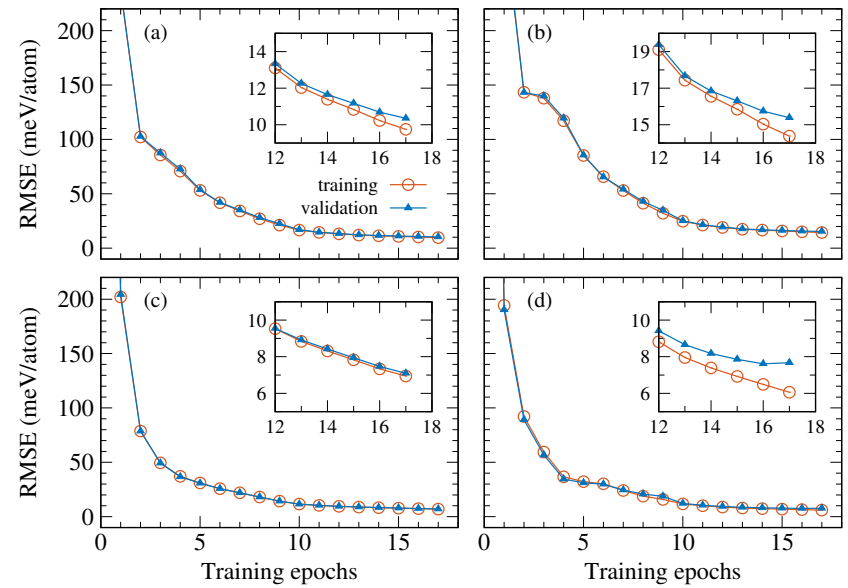

Figure 5: The convergence of the RMSE values with respect to the number of training epochs for (a) all, (b) bulk, (c) cluster, and (d) stoichiometric (bulk and cluster) structures. Note that difference in the RMSE between the training and the validation data is small, in the range of $1 \mathrm{meV} /$ atom.

In order to evaluate the accuracy of the CENT MgO potential we study the interatomic distances at finite temperatures by performing MD simulations both with CENT and DFT for a supercell containing 64 atoms. We use a Nose-Hoover thermostat to model a canonical ensemble. Fig. 6 shows the comparison of the radial distribution functions using CENT and DFT as averaged over the MD trajectories at temperatures of $300 \mathrm{~K}$ and $1000 \mathrm{~K}$ after sufficient equilibration. The overall agreement between the CENT and DFT results is good, especially in the low-temperature regime where the atomic excursions from their equilibrium positions is rather small.

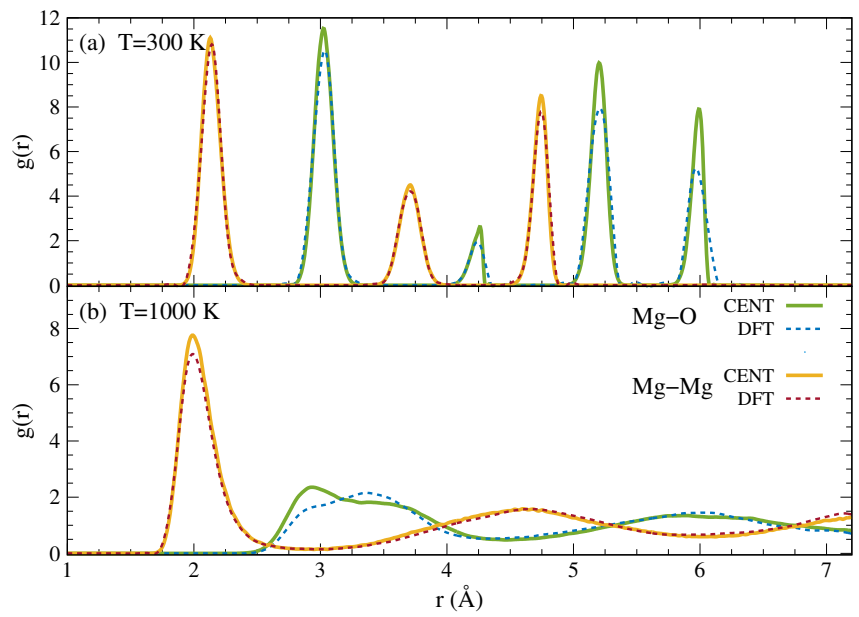

Figure 6: The radial distribution functions of the of $\mathrm{Mg}-\mathrm{O}$ and $\mathrm{Mg}-$ $\mathrm{Mg}$ interactions computed with CENT and DFT at finite temperatures of (a) $T=300 \mathrm{~K}$ and (b) $T=1000 \mathrm{~K}$. 
Table 1: Detailed description of the DFT reference data.

\begin{tabular}{cccccccc}
\hline \hline & \multicolumn{3}{c}{ Training data set } & & \multicolumn{3}{c}{ Validation data set } \\
\cline { 2 - 3 } Composition & Cluster & Bulk & Total & & Cluster & Bulk & Total \\
\hline Stoichiometric & 8,518 & 1,192 & 9,710 & & 1,787 & 248 & 2,035 \\
Non-stoichiometric & 8,521 & 5,949 & 14,470 & & 1,863 & 1,350 & 3,213 \\
Total & 17,039 & 7,141 & 24,180 & & 3,650 & 1,598 & 5,248 \\
\hline \hline
\end{tabular}

\section{Exploring Potential Energy Surfaces}

\subsection{Local Geometry Optimization}

Local minima on the PES correspond to dynamically stable configurations and define the structural geometry of stable and metastable molecules and crystalline polymorphs. Various algorithms to identify such minima in the vicinity of a starting configuration are implemented in FLAME, ranging from the simple gradient descent approach to quasi-Newton methods and damped dynamics.

The steepest descent (SD) with energy feedback is the most reliable and numerically stable method available in FLAME. In addition to the common termination conditions based on either the maximum number of iterations or a force tolerance, FLAME offers an additional criterion based on the energy saturation. The latter termination mode is especially suitable when using SD as a robust precursor to subsequent optimizers that are more efficient but only perform reliably within a quadratic region of the PES.

The conjugate gradient (CG) method is implemented in conjunction with a line search based on a quadratic approximation. Although the CG converges faster than SD in general, each optimization step requires two force evaluations. Two flavors of quasi-Newton methods are available in FLAME, namely the stabilized quasi-Newton minimizer (SQNM) 42 and the Broyden-Fletcher-Goldfarb-Shanno (BFGS) method [43, 44] with different types of (approximate) line searches. Although quasi-Newton methods are very efficient they tend to fail if the initial structure is not in the vicinity of a local minimum. The fast inertial relaxation engine 45] (FIRE) is an efficient method based on damped dynamics and presents a good compromise between reliability and efficiency. Its implementation in FLAME works particularly well for variable cell shape relaxation with and without constraints.

\subsection{Structure Prediction}

The task in structure prediction is to identify the lowest energy state on the PES, the global minimum, at given conditions. Due to the high dimensionality of the PES, the search for this ground state presents a formidable task, especially for large systems with many degrees of freedom. In particular, the curse of dimensionality leads to an exponential increase of the number of local minima on the PES with respect to the number of atoms in the system [19, which renders the search for the global minimum extremely challenging. In fact, it is impossible to exhaustively map out all minima for any realistic system, and sophisticated sampling methods are called for to efficiently and thoroughly explore the relevant, low-lying portions of a PES.

\subsubsection{Minima Hopping Method}

The minima hopping method [46 48] implements a global optimization algorithm which has proven to be particularly robust and reliable. In FLAME, the keyword task: minhocao (Minima Hopping for Crystal Optimization) in the main block triggers a MHM run. The MHM employs a sequence of successive short MD runs and geometry relaxations to "hop" between local minima, combined with several levels of sophisticated feedback mechanisms to learn the features of the PES. Within each hop, the MHM attempts to escape from the current local minimum, $M_{\text {cur }}$, using a short MD simulation with a predefined kinetic energy, followed by a local geometry relaxation. If the escape trial fails, the kinetic energy $E_{\text {kin }}$ is slightly increase (commonly by around $2-5 \%$ ) to improve the chances to escape in a the next MD escape step. On the other hand, if the escape trial succeeds, the kinetic energy is slightly reduced. This continuous adaption of $E_{\text {kin }}$ is the first feedback mechanism.

The importance of reducing the kinetic energy upon a successful escape lies in the Bell-Evans-Polanyi (BEP) principle 49], which states that exothermic chemical reactions have, on average, low activation barriers. Hence, a low kinetic energy during an MD simulation ensures that only low-energy barriers can be crossed (corresponding to a low activation energy) due to energy conservation, behind which one is more likely to encounter low-energy local minima (corresponding to an exothermic reaction).

A second feedback mechanism controls another energy parameter, called $E_{\text {diff, }}$ which introduces an additional preferences for hops towards low-energy structures. A successful hop to $M_{\text {new }}$ is only accepted if its energy differs from the previous local minimum by less than a positive threshold value: $E\left(M_{\text {new }}\right)-E\left(M_{\text {cur }}\right)<E_{\text {diff. }}$. The value of $E_{\text {diff }}$ is adjusted in such a way that half of all the performed MHM hops is accepted, while the rest is rejected and a new escape trial is performed, ensuring that even high-energy structures are eventually accepted after sufficiently many steps.

The final feedback mechanism is based on the history of visited local minima. If a known minimum is revisited by the MHM, the kinetic energy is significantly increased. In this way, the system is quickly pushed away from portions of the PES that have already been sampled, towards new, unexplored regions. In fact, the intricate interplay 
between the parameters $E_{\text {kin }}$ and $E_{\text {diff }}$ ensures that an MHM simulation will never get stuck on any part of the PES: after a funnel has been explored, the kinetic energy will start to increase due to the feedback on $E_{\text {kin. Ini- }}$ tially, this will lead to the sampling of high-energy local minima, which will be rejected at first due to the low value of $E_{\text {diff. }}$ However, after a while they will be accepted, and the MHM will leave a funnel (superbasin) to explore new portions of the PES. The interplay between the different feedback mechanisms is illustrated in Fig. 7.

The feedback mechanisms above require a reliable method to compare local minima. In FLAME, this comparison is performed based on a combination of energy differences and structural fingerprints that provide a similarity metric. A detailed description of the available methods in FLAME can be found in Sec. 5. Overall, the collection of feedback mechanisms is an essential part of the MHM which differentiates it from thermodynamics based approaches like Simulated Annealing or Basin Hopping.

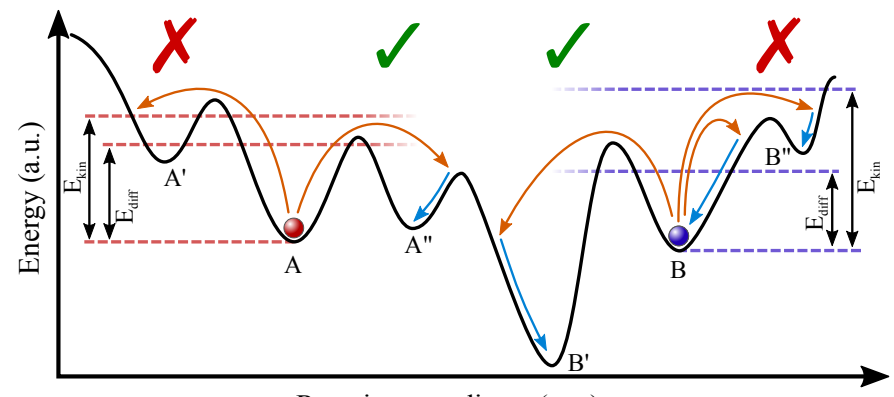

Reaction coordinate (a.u.)

Figure 7: A schematic illustration of the working principles of the MHM. The solid black line represents a 1D model energy landscape with its local minima and saddle points. The values of the two feedback quantities $E_{\text {kin }}$ and $E_{\text {diff }}$ determine if a hop is possible, and if it is accepted. The hop from A to $\mathrm{A}^{\prime}$ is prohibited due to the lower value of $E_{\mathrm{kin}}$ compared to the barrier separating the two minima, while the hop to A" is allowed and preferred. The hop from $\mathrm{B}$ to $\mathrm{B}$ " is rejected since the energy difference of the two local minima exceeds the value of $E_{\text {diff }}$, while the hop to B' is allowed and leads the system to its ground state.

\subsubsection{Softening}

Since the MHM hops between the local minima are essentially chemical reactions, they correspond to rare events that occur on a much longer time scale than observable through conventional MD simulations. In fact, the system will frequently merely oscillate in the catchment basin surrounding a local minimum during an MD escape trial. To accelerate the escape from a local minimum, the MD trajectories must be biased towards a transition into a neighboring catchment basin. Hence, we project out the velocity components along hard modes to essentially "shoot" the system preferably into a direction of low curvature. This procedure, which is called "softening", accelerates the structural search due to the following reasons.
- By eliminating the high-frequency vibrations we can use larger time steps to integrate the equations of motion for longer time scales, which directly reduces the computational cost.

- We can better exploit the BEP: there is a correlation between the curvature of the eigenmodes in a local minimum and the height of the transition barrier encountered in that direction. Hence, starting an MD simulation approximately along a soft direction increases the probability of encountering a low barrier, behind which one is more likely to find a low-energy structure. 50]

FLAME implements a dimer method to identify the low curvature direction at a local minimum. Initially, the direction of a dimer of length $d$ is chosen by randomly generating an initial velocity vector based on a Boltzmann distribution with the current kinetic energy $E_{\text {kin }}$. This velocity vector is iteratively rotated by minimizing the dimer energy with a gradient descent method, until the curvature along the dimer direction is sufficiently low or if a predefined amount of softening iterations is reached. This softening procedure is repeated for every MHM step prior to performing the MD escape trial. In FLAME, the dimer length $d$ as well as the step size within the dimer minimization can be set manually, or a gradient feedback mechanism can be used to automatically adjust their values.

The progress of the softening procedure can be monitored in the output of FLAME, where the dimer energy is reported together with the curvatures along the dimer direction. Two approximations of the curvatures are used, either based on the second order finite difference of the energies or from the first order finite difference based on the force acting on the dimer. Both approximations will converge to the same value in the limit of small dimer size $d$ within the harmonic approximation.

The number of softening iterations should be large enough to eliminate the high-frequency vibration, but not too large since the dimer will converge to point exactly along the lowest curvature eigenmode, thereby sacrificing ergodicity. As a rule of thumb, we have found that reducing the initial curvature $\kappa_{0}$ by one order of magnitude is optimal, and the softening should be stopped as soon as $\kappa_{i}<0.1 \kappa_{0}$.

\subsubsection{Example}

The MHM has been used in the past to predict the structure of a wide range of materials, predominantly of inorganic compounds [37, 51, 62. However, the implementation of the MHM in FLAME includes a wide range of $\mathrm{BC}$ and constraints that can be tuned to optimize the search for any type of material. E.g., individual components of the simulation cell $\{a, b, c, \alpha, \beta, \gamma\}$ can be constrained if experimental lattice parameters are known, or 2-dimensional 
confinement potentials can be included to model $2 \mathrm{D}$ materials or layered structures $37,63,64$. Here, we demonstrate the use of FLAME to predict the structure of a molecular, organic crystal, and show how the various parameters evolve during the course of a search in Fig. 8 .

We aim to predict the ground state of Formaldehyde, a simple molecule with the chemical formula $\mathrm{CH}_{2} \mathrm{O}$. Experimentally, the ground state structure of Formaldehyde and its deuterated version has been resolved at $15 \mathrm{~K}$ by Weng et al. 65. Hence, for this particular case, we know what the solution of our search problem is. The crystal structure of the ground state has $P \overline{4} 21 c$ symmetry (space group index 114), and its unit cell contains 8 f.u. To model the atomic interaction in FLAME, we employ a semiempirical method, the density functional tight binding method as implemented in the DFTB + package [31. We include Van der Waals interactions in our PES, modeled by empirically fitted pairwise potentials, and employ a sufficiently dense k-point sampling to converge the total energy.

The initial seed structure for our MHM is generated randomly, and is shown in the bottom panel of Fig. 8, inset (a). This structure is quite high in energy with respect to the ground state at above $120 \mathrm{meV} /$ f.u., so we can assume that it is rather far away from the ground state structure also in configurational space. We further initialize the two main feedback parameters, $E_{\text {kin }}$ and $E_{\text {diff }}$, with conservative values of $100 \mathrm{~K}$ and $2.5 \mathrm{meV} /$ f.u., respectively. Note that we give the kinetic energy here in units of a temperature (an intensive quantity), but it does not correspond to a real temperature since (a) the MD escape trials are very short bursts solely intended to overcome reaction barriers and (b) the relative masses of the atoms are scaled to reduce the range of the frequency spectrum stemming from all vibrational modes.

Since we are dealing with a molecular crystal we have to preserve the molecular units and avoid dissociation of the individual molecules. One way to impose this constraint is to treat each molecule as a rigid object, thereby significantly decreasing the degree of freedom (DOF): every molecule has 6 DOF (3 rotational and 3 translational) if treated as a rigid body, whereas there are $3 \times N_{\text {Nat }}=12$ DOF if we take into account all internal coordinates in $\mathrm{CH}_{2} \mathrm{O}$. Here, we choose an alternative method, namely projecting out the strong intramolecular vibrations in the MD escape trials by performing a relatively large, fixed number of softening steps for the atomic degrees of freedom, nsoften $=40$. In this way, the kinetic energy will be naturally distributed to perform molecular or global moves in the configurational space without breaking any intramolecular bonds.

Within the first few dozen MHM steps, the energy of the explored local minima decreases rapidly (blue crosses in the bottom panel of Fig. 8), many of which are accepted (dark blue circles). This is the most favorable behavior of the MHM, and rapid progress is made in exploring new, low-energy local minima. During this part of the MHM, the parameters $E_{\text {kin }}$ and $E_{\text {diff }}$ do not change significantly,

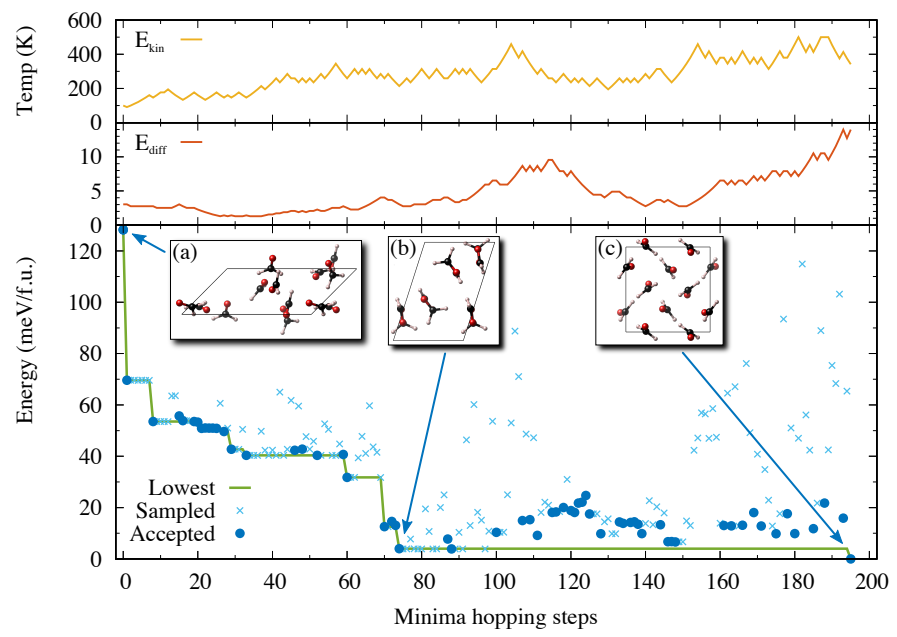

Figure 8: The evolution of the parameters during a serial MHM structure prediction run on Formaldehyde. The top and middle panels shows the kinetic energy for each of the MD escape steps and the feedback parameter $E_{\text {diff }}$, respectively. The bottom panel shows the evolution of the potential energy of the explored local minima. The light blue crosses denote all visited minima, while the dark blue filled circles denote the accepted local minima. The green line represents the lowest energy structure found up to a given MHM iteration.

simply fluctuating slightly around some equilibrium value, as shown in the top two panels of Fig. 8. This behavior changes after reaching step 75 in the MHM, when a very favorable structure with nearly ideal molecular packing is found (inset (b) in Fig. 8). For the next $\approx 100$ steps, no lower energy minimum is found, and the simulation is stuck in a large funnel of the PES. As expected, the feedback on $E_{\text {kin }}$ and $E_{\text {diff }}$ kicks in, and their values start to increase, thereby driving the system towards new, unexplored regions of the PES. Eventually, after visiting 196 structures, the MHM algorithm escapes from the "wrong" funnel and successfully finds the ground state, shown in inset (c) of Fig. 8 .

\subsection{Transition State Searches}

Transition states or saddle points are stationary points of the PES at which the Hessian matrix has all but one positive eigenvalues. Saddle points on the PES are important since they determine the kinetics of chemical reactions and phase transformations according to transition state theory 66]. In fact, two neighboring local minima on a PES can be connected through the MEP, along which a saddle point always denotes the state of highest energy and determines the reaction rate of this particular transition. Identifying a saddle point on a PES is overall more challenging than finding a local minimum, since there is no associate target function that can be optimized by rigorously following its gradients.

Transition state search methods are classified into two types: (i) methods that identifies a saddle point close to an initial configuration on the PES, thereby neglecting which minima it connects, and (ii) methods that aim at finding a saddle point connecting two known, usually 
neighboring, local minima, thereby also attaining the associated MEP. In this manuscript, we denote types (i) and (ii) as one-sided and two-sided methods, respectively. Typically, one-sided methods follow the minimum mode by inverting the gradient component, in an approximate manner, along the eigenvector associated with the lowest eigenvalue. The dimer method 67] is a prominent example of this type, while the nudged elastic band (NEB) equipped with the climbing-image approach 68] represents a two-sided method. Both above methods are available in FLAME, together with two additional techniques that are discussed in detail below.

\subsubsection{Enhanced Splined Saddle Method}

The main issue that prevents a smooth convergence towards a saddle point between two minima is the absence of a target function to optimize. The splined saddle method 69] developed by Granot and Baer, and later improved by Ghasemi and Goedecker [70, alleviates this issue by mapping the problem onto convex optimization task. It splits the saddle point search into two nested optimization tasks where the inner loop is a one-dimensional global maximization along the pathway that is embedded in the outer loop which minimizes the maximum point obtained in the inner loop. The method utilizes splines to characterize the pathway, hence the energy of the maximum point (EMP) along the path is a function of the coordinates of anchor points. The gradient of the EMP with respect to the anchor points can thus be calculated using the equations given in Ref. 70] and its appendix. With the gradient of the target function at hand, any gradientbased optimizer can be directly employed to find its extremum. Critical to this method is the confidence in finding the global maximum along the pathway which in turn can severely affect the reliability of the target function and its gradient. As shown in Ref. [70, the method can be very efficient and outperform competing algorithms. However, the method may be unstable for long pathways, in particular when there exists an intermediate minimum along the reaction trajectory.

\subsubsection{Bar-Saddle Method}

The bar-saddle method [71] is primarily suited for one-sided searches and is a modification of the dimer method 67. A bar (or dimer) is moved on the PES in such a way that its center converges towards a saddle point while its orientation points along the direction of lowest (negative) curvature. To achieve this, the forces acting on its end points are decomposed and used to iteratively rotate and translate the bar, eventually moving its center towards the saddle point.

The main difference between the bar-saddle approach and the dimer method lies in how the rotational and translational forces are computed. Within the dimer method the rotation is expressed in terms of the rotational angle $d \theta$ within the plane of rotation, given the force acting perpendicular to the dimer. In contrast, the bar-saddle

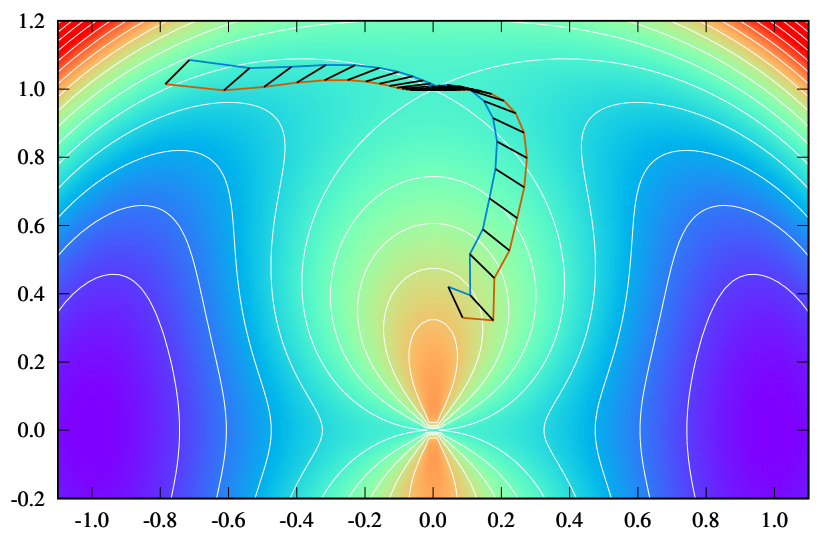

Figure 9: Trajectory of the barsaddle method starting from two different initial positions on a model energy landscape $f(x, y)=(1-$ $\left.\left(x^{2}+y^{2}\right)\right)^{2}+\left(y^{2}\right) /\left(x^{2}+y^{2}\right)$. The two local minima (dark purple) are located at $( \pm 1,0)$, and the saddle point is located at $(0,1)$.

approach applies a rotational force on the two endpoints of the bar, $A$ and $B$, according to $\mathbf{F}_{A}^{\text {Rot }}=\frac{1}{2}\left(\mathbf{F}_{A}^{\perp}-\mathbf{F}_{B}^{\perp}\right)$ and $\mathbf{F}_{B}^{\text {Rot }}=\frac{1}{2}\left(\mathbf{F}_{B}^{\perp}-\mathbf{F}_{A}^{\perp}\right)$, where $\mathbf{F}_{\mathrm{i}}^{\perp}=\mathbf{F}_{\mathrm{i}}-\mathbf{F}_{\mathrm{i}}^{\|}$are the force components perpendicular to the bar ends. For the translational forces, the dimer method uses the inverted gradient component along the dimer, averaged over the two endpoints. The bar-saddle method on the other hand uses a cubic interpolation to estimate the force acting along the bar, thereby assuming that the negative mode at the saddle point is harmonic. After moving the bar using the combination of rotational and translational forces, the bar length is rescaled to its target value, $d_{b a r}$.

Since the bar-saddle method is implemented as a onesided approach in FLAME, the initial position of the bar has to be provided as an input and should be ideally in the vicinity of a saddle point. The dimer is iteratively optimized using a gradient feedback method. Additionally, the bar size can be contracted towards the end of a search. In this way, the saddle point search starts out with a rather long bar to approximately locate the saddle point, and hones in on its exact location as the gradients on the bar decreases and the search converges. Fig. 9 shows a model PES together with the iterative steps of the barsaddle method as it locates the saddle point, starting from two initial configurations.

\subsubsection{Example}

To demonstrate the saddle point optimization in FLAME, we study the diffusion of an oxygen vacancy near the surface of a $\mathrm{MgO}$ slab. Two of the diffusion mechanisms, one towards the surface $\left(\mathrm{O}^{1}\right)$ and the other towards the bulk region $\left(\mathrm{O}^{2}\right)$, are shown in Fig. 10 . The oxygen vacancy moving to the surface of the slab passes over a barrier that is by $\approx 0.7 \mathrm{eV}$ lower compared to the diffusion towards the bulk. However, both processes in- 
volve crossing high barriers which may not be surmounted at room temperature in time scales occurring in typical experiments. The splined saddle method is used in these calculations which can be invoked by setting the task key in the main block to saddle and method key to splined_saddle in the saddle block.

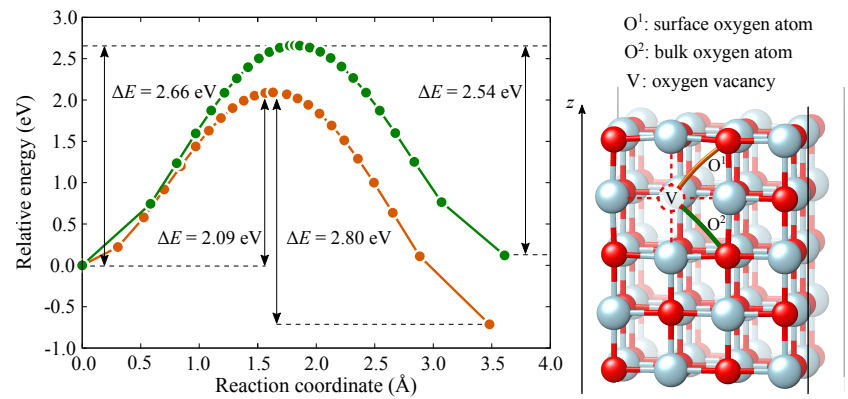

Figure 10: Oxygen vacancy diffusion barriers computed with the enhanced splined saddle method. The left panel shows the energy along the MEP for the diffusion of the vacancy towards the surface and the center of the bulk (opposite $z$ directions). The right panel shows the model slab structure used in our calculation. The surface normal points along the $z$ direction.

\section{Atomic Environment Descriptors and Struc- tural Fingerprints}

Structural descriptors of a system or the local environment of its components, the atoms, have to be translationally and rotationally invariant. Further, atomic environment descriptors have to be invariant under the permutation of the atoms. Hence, Cartesian coordinates are not well suited, and alternative schemes are called for to feed the input layers of an ANN potential, or to define a metric in configurational space to compare structural differences.

A common approach is to map the Cartesian coordinates into a so-called constant-sized vectorial environment descriptor, which fulfills all required symmetry conditions. Behler introduced a suitable environment descriptor, the atom-centered symmetry functions (ACSFs) 72, which are constructed by summing up smooth two-body and three-body functions. The former gives information on radial distribution surrounding each atom within a localization region, while the latter provides also information about the angular arrangements. Several other descriptors, e.g., the smooth overlap of atomic positions [73] (SOAP) by Bartók et al. or the overlap matrix (GOM) [74, 75. by Li et al. have been developed, and the latter is available in the FLAME code.

The above descriptors can also be used as structural fingerprints to define a metric $d(a, b)$ in configurational space and to compare two different structures $a$ and $b$. Since the distance between these two structures must be invariant under the exchange of any two atoms in the respective structures, the distance must be minimized over all possible permutations $P$ that matches atom $k$ in $a$ with
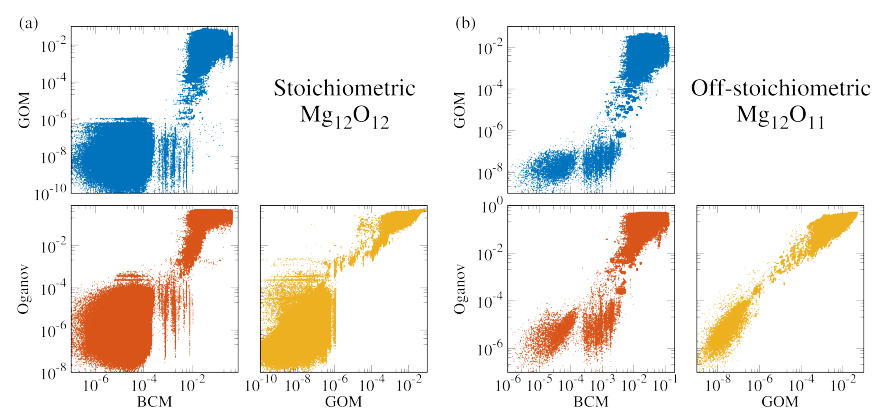

Figure 11: Pairwise distances using three different structural fingerprints on two periodic $\mathrm{Mg}-\mathrm{O}$ system. The Oganov and $\mathrm{BCM}$ are global fingerprint methods, while the GOM constitutes an atomicbased approach. The units on the axis are arbitrary.

atom $P(k)$ in b:

$$
d(a, b)=\min _{P} \sum_{k}^{N} g\left(\mathbf{V}_{k}^{a}, \mathbf{V}_{P(k)}^{b}\right)
$$

where $\mathbf{V}_{i}^{x}$ is the atomic environment vector of atom $i$ in structure $x$, and $g(\mathbf{p}, \mathbf{q})$ is a norm defined on the individual atomic environment descriptors $\mathbf{p}$ and $\mathbf{q}$. The ideal permutation is found using the Hungarian algorithm with cubic scaling.

In contrast to such atomic-based methods, global fingerprints integrate out the atomic contributions to give a single descriptive vector for a structure. Such global methods come at a loss of information, but are often faster to compute since the minimization with respect to atomic permutations can be omitted. Structural comparison algorithms can become a bottleneck for global optimization tasks using classical force fields, where thousands of structures are rapidly sampled and have to be efficiently compared. In FLAME, the currently implemented global fingerprints include the Oganov method [76] and the bond characterization matrix (BCM) [77, 78].

\subsection{Example}

We compare three different environmental descriptor metrics by analyzing their performance on two periodic systems, $\mathrm{Mg}_{12} \mathrm{O}_{12}$ and the off-stoichiometric $\mathrm{Mg}_{12} \mathrm{O}_{11}$. For both systems we generate a wealth of candidate structures using the MHM and the CENT potential. Using several random input structures, we perform a total of around 1,500 MHM hops for $\mathrm{Mg}_{12} \mathrm{O}_{12}$ and $\mathrm{Mg}_{12} \mathrm{O}_{11}$. Note that we include both the accepted and rejected local minima of the MHM runs as well as all failed escape trials, thereby including many potential duplicate structures. In FLAME, the fingerprints and their distances can be computed on-the-fly during a simulation, or as a post-processing task for data filtering and pruning. Hence, we a posteriori compute the fingerprint distances between every pair of structures with respect to the Oganov [76], BCM [77, 78], and GOM [74, 75] method. 
In Fig 11 we show all possible combinations of the pairwise distances for the two data sets, where different fingerprint methods are plotted along the $x$ and $y$ axis. Panels (a) and (b) correspond to $\mathrm{Mg}_{12} \mathrm{O}_{12}$ and $\mathrm{Mg}_{12} \mathrm{O}_{11}$, respectively. Ideally, two fingerprints that perform similarly would correlate well and produce a diagonal line, with a clear gap separating structures that are classified as identical and distinct. For the $\mathrm{Mg}_{12} \mathrm{O}_{12}$ system we clearly see that all three fingerprints identify a large number of structures that are identical, shown be the large points cloud at the bottom left. This large region corresponds primarily to rock-salt structures and slightly defective versions thereof, indicating that $\mathrm{Mg}-\mathrm{O}$ is a strong structure seeker with well-defined global minimum. A close analysis of the subplots shows that there are however some subtle differences between the three methods. E.g., while the GOMOganov subplot exhibits a rather clear correlation, both BCM-Oganov and BCM-GOM shows that the BCM has trouble classifying identical structures based on the several sub-clusters, showing up as vertical lines, without a clear gap.

The correlation plots for $\mathrm{Mg}_{12} \mathrm{O}_{11}$ looks slightly different with less pronounced points clouds at the bottom left. Indeed, the PES of the non-stoichiometric $\mathrm{Mg}_{12} \mathrm{O}_{11}$ system is more complex with a less clearly defined ground state. Similar to $\mathrm{Mg}_{12} \mathrm{O}_{12}$, the correlation between GOM and Oganov fingerprint is clearly visible, while the BCM exhibits a rather continuous fingerprint spectrum with ill defined clusters. These results are in good agreement with the findings of Zhu et al. [75, who performed extensive tests comparing structural difference metrics across various systems. In practice, the GOM or Oganov fingerprint metrics are a good choice for production runs in FLAME, especially due to their much lower computational cost compared to the BCM method.

\section{Electrostatic Interactions}

An important ingredient for the CENT method is the calculation of the electrostatic Hartree term. This can be done either by using one of the methods implemented in FLAME [79, 80, or by relying on one of the external solvers implemented in the BigDFT PSolver [1-4] library. BigDFT PSolver employs appropriate Green's function depending on the BC to solve the Poisson's equation and therefore avoids introducing any artifact, with a favorable scaling behavior of $\mathcal{O}(N \log (N))$. Depending on the BC, the available solvers in FLAME are as follow.

Free BC The simplest electrostatic method implemented in FLAME is the pairwise summation according to atomic charge densities. The method scales $\mathcal{O}\left(N^{2}\right)$ and is only suited for small systems. For larger systems we recommend using the quasi-linear scaling methods from the BigDFT PSolver library [2].

Slab BC Systems with (quasi-)two-dimensional slablike geometries are typically modeled with periodic $\mathrm{BC}$ in two dimensions and free $\mathrm{BC}$ in the third, referred to as slab BC in this manuscript. The $\mathrm{P}^{3} \mathrm{D}$ method 79 , implemented in FLAME, solves the Poisson's equation while correctly dealing with such a slab BC. Consequently, no vacuum region is required in the direction perpendicular to the surface, in contrast to standard plane wave based Poisson solvers with fully three-dimensional periodicity. Therefore, highly accurate results can be obtained even in the presence of non-vanishing dipole moments along the surface direction. In particular, the CENT potential in FLAME can be used to study polar surfaces of materials and their properties. Also, the $\mathrm{P}^{3} \mathrm{D}$ method scales as $\mathcal{O}(N \log (N))$ where $N$ is the number of particles in the simulation cell. In the $\mathrm{P}^{3} \mathrm{D}$ method, both the charge density and potential are expanded in plane waves in the two periodic directions, while using finite elements in the third dimension.

Bulk BC The Hartree energy for three-dimensional, fully periodic systems in the CENT potential can be calculated either by Fourier summation or by the BigDFT PSolver. The Fourier summation is calculated using

$$
\begin{aligned}
U^{\mathrm{es}}= & \frac{2 \pi}{V} \sum_{\mathbf{k} \neq 0} \frac{1}{\mathbf{k}^{2}}\left[\left(\sum_{i=1}^{N} q_{i} \cos \left(\mathbf{k} \mathbf{R}_{i}\right) \exp \left(-\frac{\alpha_{i}^{2} \mathbf{k}^{2}}{4}\right)\right)^{2}\right. \\
& \left.+\left(\sum_{i=1}^{N} q_{i} \sin \left(\mathbf{k} \mathbf{R}_{i}\right) \exp \left(-\frac{\alpha_{i}^{2} \mathbf{k}^{2}}{4}\right)\right)^{2}\right],
\end{aligned}
$$

where $V$ is the volume of the simulation cell, $q_{i}$ are the atomic charges, and $\alpha_{i}$ are the widths of the Gaussian atomic charge densities.

The stress tensor in CENT is the sum of the short range part due to the environment dependent atomic electronegativities, and the long range part from the Hartree energy. The former is calculated with

$$
\begin{aligned}
& \sigma_{\alpha \beta}^{\text {short }}=\sum_{i, j=1}^{N} R_{i j}^{\alpha} F_{i j}^{\beta} \\
&=-\sum_{k=1}^{N} \sum_{l=1}^{N_{k}} q_{k} \frac{\partial \chi_{k}}{\partial G_{l}^{k}} \sum_{i, j=1}^{N} R_{i j}^{\alpha} \frac{\partial G_{l}^{k}}{\partial R_{j}^{\beta}}
\end{aligned}
$$

where $R_{i j}^{\alpha}$ is the component $\alpha$ of $\mathbf{R}_{i j}=\mathbf{R}_{j}-\mathbf{R}_{i}$, and $F_{i j}^{\beta}$ is the component $\beta$ of the force applied on atom $i$ by atom $j . N_{k}$ is the number of symmetry functions, indeed the number of nodes in the ANN input layer. $G_{l}^{k}$ is $l$-th element of symmetry function array of atom $k$. The latter contribution to the stress tensor is calculated using the derivatives of the Hartree energy,

$$
\sigma^{\mathrm{es}}=-\frac{1}{V} \frac{\partial U^{\mathrm{es}}}{\partial h} h^{T} .
$$




\subsection{Example}

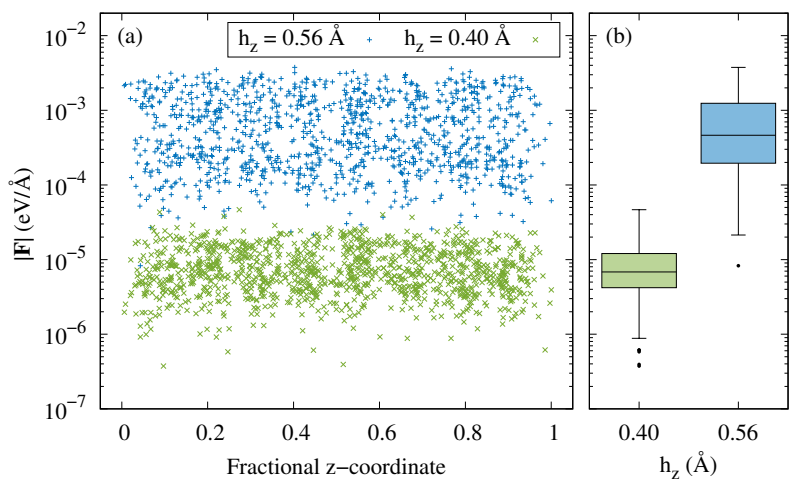

Figure 12: Norms of the force errors for each of the atoms with the CENT method within a slab geometry calculated by the $\mathrm{P}^{3} \mathrm{D}$ method. Due to the proper treatment of $\mathrm{BC}$, there is no sign dependency in the force errors along the direction orthogonal to the surface of the slab. Also, it is shown that a small decrease in grid spacing can reduce the error by more than two orders of magnitude.

To demonstrate the accuracy of the $\mathrm{P}^{3} \mathrm{D}$ method in evaluating the electrostatic interaction, we present here an analysis of its errors when used in conjunction with the CENT potential. More precisely, we investigate how the accuracy of the atomic forces behaves as a function of the grid density in the $\mathrm{P}^{3} \mathrm{D}$ scheme for a slab of $\mathrm{MgO}$. To recall, in the CENT potential the charge density is given by a superposition of atom-centered smooth Gaussian charges.

In order to gather statistically significant results we generate a set of slab structures by performing an MD simulation at $1000 \mathrm{~K}$, starting with a rocksalt-like structure consisting of 100 atoms. We then randomly select 12 distinct MD snapshots, which serve as our benchmark structures. To generate the reference data we compute the forces acting on each atom using fine grid spacings of $h_{x}=h_{y}=0.29 \AA$ in the two periodic dimensions, and $h_{z}=0.26 \AA$ along the non-periodic, out-of-plane direction. With these tight settings the forces are converged to essentially within machine precision.

To assess the accuracy of the $\mathrm{P}^{3} \mathrm{D}$ method we then recompute the atomic forces using larger values of $h_{z}$ while keeping $h_{x}=h_{y}=0.29 \AA$ fixed. For most CENT calculations, a spacing of $h_{z}=0.56 \AA$ is sufficiently small to give reliable results of standard accuracy. The blue crosses in Fig. 12 show the error in the force norms on each atom as a function of the $z$-components for all $12 \times 100$ atoms. Here, the units of $z$ is given in fractional coordinates with respect to $z_{\min }$ and $z_{\max }$ of the two outmost atoms at the top and bottom of the slabs, respectively. Note that there is no systematic pattern in the error distribution along the $z$ direction, indicating that the error in atomic forces at the surfaces and at the center of the slab are virtually identical. Such a behavior is crucially important when dealing with surfaces and interfaces, and our results demonstrate that the $\mathrm{P}^{3} \mathrm{D}$ method is particularly well suited to handle these systems.
If a higher accuracy is required, a slight decrease in the grid spacing is sufficient: By reducing the value of $h_{z}$ to $0.40 \AA$, the errors in the force norms drops by two orders of magnitude at only a moderate increase in computational cost, as shown by the green crosses in Fig. 12. However, for most practical applications there is no need to go beyond this level of accuracy.

\section{Conclusions}

The use of ML techniques for atomistic simulations is becoming increasingly popular, and their deployment in interatomic potentials can significantly accelerate and improve theoretical predictions. The FLAME code implements the CENT ANN potential together with a plethora of state-of-the art atomistic modeling techniques in a fully integrated open-source software package. When compiled as a library, the CENT potential can be used as a blackbox engine and used by third-party software packages, like LAMMPS. On the other hand, the sampling algorithms within FLAME can readily be linked with external (quantum) engines, like LAMMPS, VASP, ABINIT, Quantum ESPRESSO, and many more. In fact, FLAME can act as a server and communicate over sockets with any package that supports the i-Pi protocol, which has been meanwhile integrated in a range of codes.

The seamless integration of the CENT potential with the MHM for structure prediction is a particularly powerful feature of FLAME. The unique combination of a rapid PES exploration scheme with an efficient and accurate interatomic potential has proven to be especially valuable in materials discovery.

\section{Acknowledgements}

We thank Luigi Genovese, Thomas Lenosky, and Stefan Goedecker for fruitful discussions. M.A. acknowledges support from the Novartis Universität Basel Excellence Scholarship for Life Sciences and the Swiss National Science Foundation (projects P300P2-158407, P300P2174475, and P4P4P2-180669).

\section{References}

[1] L. Genovese, A. Neelov, S. Goedecker, T. Deutsch, S. A. Ghasemi, A. Willand, D. Caliste, O. Zilberberg, M. Rayson, A. Bergman, R. Schneider, J. Chem. Phys. 129 (2008) 014109.

[2] L. Genovese, T. Deutsch, A. Neelov, S. Goedecker, G. Beylkin, J. Chem. Phys. 125 (2006) 074105.

[3] A. Neelov, S. A. Ghasemi, S. Goedecker, J. Chem. Phys. 127 (2007) 024109.

[4] L. Genovese, T. Deutsch, S. Goedecker, J. Chem. Phys. 127 (2007) 054704.

[5] A. Togo, I. Tanaka, arXiv:1808.01590 [cond-mat] (2018). ArXiv: 1808.01590.

[6] F. H. Stillinger, T. A. Weber, Phys. Rev. B 31 (1985) 5262.

[7] J. Tersoff, Phys. Rev. B 39 (1989) 5566.

[8] J. F. Justo, M. Z. Bazant, E. Kaxiras, V. V. Bulatov, S. Yip, Phys. Rev. B 58 (1998) 2539. 
[9] T. J. Lenosky, B. Sadigh, E. Alonso, V. V. Bulatov, T. D. de la Rubia, J. Kim, A. F. Voter, J. D. Kress, Modelling Simul. Mater. Sci. Eng. 8 (2000) 825.

[10] A. C. T. van Duin, S. Dasgupta, F. Lorant, , W. A. G. III, J. Phys. Chem. A 105 (2001) 9396.

[11] J. Yu, S. B. Sinnott, S. R. Phillpot, Phys. Rev. B 75 (2007) 085311.

[12] T. Liang, Y. K. Shin, Y.-T. Cheng, D. E. Yilmaz, K. G. Vishnu, O. Verners, C. Zou, S. R. Phillpot, S. B. Sinnott, A. C. T. van Duin, Annu. Rev. Mater. Res 43 (2013) 109.

[13] J. Behler, M. Parinello, Phys. Rev. Lett 98 (2007) 146401.

[14] A. P. Bartók, M. C. Payne, R. Kondor, G. Csányi, Phys. Rev. Lett. 104 (2010) 136403.

[15] M. Rupp, A. Tkatchenko, K.-R. Müller, O. A. von Lilienfeld, Phys. Rev. Lett. 108 (2012) 058301.

[16] A. P. Thompson, L. P. Swiler, C. R. Trott, S. M. Foiles, G. J. Tucker, J. Comput. Phys. 285 (2015) 316-330.

[17] S. A. Ghasemi, A. Hofstetter, S. Saha, S. Goedecker, Phys. Rev. B $92(2015) 045131$.

[18] A. P. Bartók, G. Csányi, Int. J. Quantum Chem. 115 (2015) 1051-1057.

[19] F. H. Stillinger, Phys. Rev. E 59 (1999) 48-51.

[20] A. R. Oganov, Modern Methods of Crystal Structure Prediction, Wiley, 1 edition, 2010.

[21] A. Khorshidi, A. A. Peterson, Comput. Phys. Commun. 207 (2016) 310-324.

[22] A. S. Abbott, J. M. Turney, B. Zhang, D. G. Smith, D. Altarawy, H. F. Schaefer, J. Chem. Theory Comput. (2019).

[23] K. Yao, J. E. Herr, D. W. Toth, R. Mckintyre, J. Parkhill, Chem. Sci. 9 (2018) 2261-2269.

[24] S. Plimpton, J. Comput. Phys. 117 (1995) 1-19.

[25] A. H. Larsen, J. J. Mortensen, J. Blomqvist, I. E. Castelli, R. Christensen, M. Dułak, J. Friis, M. N. Groves, B. Hammer, C. Hargus, E. D. Hermes, P. C. Jennings, P. B. Jensen, J. Kermode, J. R. Kitchin, E. L. Kolsbjerg, J. Kubal, K. Kaasbjerg, S. Lysgaard, J. B. Maronsson, T. Maxson, T. Olsen, L. Pastewka, A. Peterson, C. Rostgaard, J. Schiøtz, O. Schütt, M. Strange, K. S. Thygesen, T. Vegge, L. Vilhelmsen, M. Walter, Z. Zeng, K. W. Jacobsen, J. Phys. Condens. Matter. 29 (2017) 273002.

[26] C. W. Glass, A. R. Oganov, N. Hansen, Comput. Phys. Commun. 175 (2006) 713-720.

[27] D. C. Lonie, E. Zurek, Comput. Phys. Commun. 182 (2011) 372-387.

[28] Y. Wang, J. Lv, L. Zhu, Y. Ma, Comput. Phys. Commun. 183 (2012) 2063-2070.

[29] C. J. Pickard, R. Needs, J. Phys. Condens. Matter. 23 (2011) 053201.

[30] M. Ceriotti, J. More, D. E. Manolopoulos, Comput. Phys. Commun. 185 (2014) 1019-1026.

[31] B. Aradi, B. Hourahine, T. Frauenheim, J. Phys. Chem. A 111 (2007) 5678-5684.

[32] S. Lorenz, A. Groß, M. Scheffler, Chem. Phys. Lett. 395 (2004) 210-215.

[33] N. Artrith, T. Morawietz, J. Behler, Phys. Rev.B 83 (2011) 153101.

[34] S. Faraji, S. A. Ghasemi, S. Rostami, R. Rasoulkhani, B. Schaefer, S. Goedecker, M. Amsler, Phys. Rev. B 95 (2017) 104105.

[35] S. Faraji, S. A. Ghasemi, B. Parsaeifard, S. Goedecker, Phys. Chem. Chem. Phys. 21 (2019) 16270.

[36] R. Rasoulkhani, H. Tahmasbi, S. A. Ghasemi, S. Faraji, S. Rostami, M. Amsler, Phys. Rev. B 96 (2017) 064108.

[37] H. A. Eivari, S. A. Ghasemi, H. Tahmasbi, S. Rostami, S. Faraji, R. Rasoulkhani, S. Goedecker, M. Amsler, Chem. Mater. 29 (2017) 8594

[38] R. Hafizi, S. A. Ghasemi, S. J. Hashemifar, H. Akbarzadeh, J. Chem. Phys. 147 (2017) 234306.

[39] S. Rostami, M. Amsler, S. A. Ghasemi, J. Chem. Phys. 149 (2018) 124106.

[40] W. J. Mortier, K. V. Genechten, J. Gasteiger, J. Am. Chem. Soc. $107(1985) 829$.
[41] I. Rivals, L. Personnaz, Neurocomputing 20 (1998) 279 - 294.

[42] B. Schaefer, S. A. Ghasemi, S. Roy, S. Goedecker, J. Chem. Phys. 142 (2015) 034112.

[43] C. G. Broyden, IMA J. Appl. Math. 6 (1970) 76-90.

[44] R. Fletcher, Comput. J. 13 (1970) 317-322.

[45] E. Bitzek, P. Koskinen, F. Gähler, M. Moseler, P. Gumbsch, Phys. Rev. Lett. 97 (2006) 170201.

[46] S. Goedecker, J. Chem. Phys. 120 (2004) 9911.

[47] M. Amsler, S. Goedecker, J. Chem. Phys. 133 (2010) 224104.

[48] M. Amsler, in: W. Andreoni, S. Yip (Eds.), Handbook of Materials Modeling: Applications: Current and Emerging Materials, Springer International Publishing, Cham, 2018, pp. 1-20.

[49] S. Roy, S. Goedecker, V. Hellmann, Phys. Rev. E 77 (2008) 056707.

[50] M. Sicher, S. Mohr, S. Goedecker, J. Chem. Phys. 134 (2011) 044106.

[51] M. Amsler, S. A. Ghasemi, S. Goedecker, A. Neelov, L. Genovese, Nanotechnology 20 (2009) 445301.

[52] S. De, S. A. Ghasemi, A. Willand, L. Genovese, D. Kanhere, S. Goedecker, J. Chem. Phys. 134 (2011) 124302.

[53] M. Amsler, J. A. Flores-Livas, L. Lehtovaara, F. Balima, S. A. Ghasemi, D. Machon, S. Pailhès, A. Willand, D. Caliste, S. Botti, A. San Miguel, S. Goedecker, M. A. L. Marques, Phys. Rev. Lett. 108 (2012) 065501.

[54] T. D. Huan, M. Amsler, V. N. Tuoc, A. Willand, S. Goedecker, Phys. Rev. B 86 (2012) 224110.

[55] T. D. Huan, M. Amsler, M. A. L. Marques, S. Botti, A. Willand, S. Goedecker, Phys. Rev. Lett. 110 (2013) 135502.

[56] R. Sarmiento-Pérez, T. F. T. Cerqueira, I. Valencia-Jaime, M. Amsler, S. Goedecker, A. H. Romero, S. Botti, M. A. L. Marques, J. Chem. Phys. 142 (2015) 024710.

[57] M. Amsler, S. Goedecker, W. G. Zeier, G. J. Snyder, C. Wolverton, L. Chaput, Chem. Mater. 28 (2016) 2912-2920.

[58] I. Valencia-Jaime, R. Sarmiento-Pérez, S. Botti, M. A. L. Marques, M. Amsler, S. Goedecker, A. H. Romero, J. Alloys Compd. 655 (2016) 147.

[59] M. Amsler, S. S. Naghavi, C. Wolverton, Chem. Sci. 8 (2017) 2226-2234.

[60] V. Balédent, T. T. F. Cerqueira, R. Sarmiento-Pérez, A. Shukla, C. Bellin, M. Marsi, J.-P. Itié, M. Gatti, M. A. L. Marques, S. Botti, J.-P. Rueff, Phys. Rev. B 97 (2018) 024107.

[61] M. Amsler, V. I. Hegde, S. D. Jacobsen, C. Wolverton, Phys. Rev. X 8 (2018) 041021.

[62] M. Amsler, Phys. Rev. B 99 (2019) 060102.

[63] M. Amsler, Z. Yao, C. Wolverton, Chem. Mater. 29 (2017) 98199828.

[64] S. Singh, Z. Zanolli, M. Amsler, B. Belhadji, J. O. Sofo, M. J. Verstraete, A. H. Romero, The Journal of Physical Chemistry Letters 0 (0) 7324-7332. PMID: 31682118.

[65] S.-X. Weng, B. Torrie, B. Powell, Mol. Phys. 68 (1989) 25-31.

[66] H. Eyring, J. Chem. Phys. 3 (1935) 107.

[67] G. Henkelman, H. Jónsson, J. Chem. Phys. 111 (1999) 7010.

[68] G. Henkelman, B. P. Uberuaga, H. Jónsson, J. Chem. Phys. 113 (2000) 9901.

[69] R. Granot, R. Baer, J. Chem. Phys. 128 (2008) 184111.

[70] S. A. Ghasemi, S. Goedecker, J. Chem. Phys. 135 (2011) 014108.

[71] B. Schaefer, S. Mohr, M. Amsler, S. Goedecker, J. Chem. Phys. 140 (2014) 9901.

[72] J. Behler, J. Chem. Phys. 134 (2011) 074106.

[73] A. P. Bartók, R. Kondor, G. Csányi, Phys. Rev. B 87 (2013) 184115.

[74] A. Sadeghi, S. A. Ghasemi, B. Schaefer, S. Mohr, M. A. Lill, S. Goedecker, J. Chem. Phys. 139 (2013) 184118

[75] L. Zhu, M. Amsler, T. Fuhrer, B. Schaefer, S. Faraji, S. Rostami, S. A. Ghasemi, A. Sadeghi, M. Grauzinyte, C. Wolverton, S. Goedecker, J. Chem. Phys. 144 (2016) 034203.

76] A. R. Oganov, M. Valle, J. Chem. Phys. 130 (2009) 104504-9.

[77] Y. Wang, J. Lv, L. Zhu, Y. Ma, Comput. Phys. Commun. 183 (2012) 2063-2070

[78] Y. Wang, J. Lv, L. Zhu, S. Lu, K. Yin, Q. Li, H. Wang, L. Zhang, Y. Ma, J. Phys.: Condens. Matter. 27 (2015) 203203. 
[79] S. A. Ghasemi, A. Neelov, S. Goedecker, J. Chem. Phys 127 (2007) 224102.

[80] S. Rostami, S. A. Ghasemi, E. Nedaaee Oskoee, J. Chem. Phys. 145 (2016) 124118. 